\title{
TRANSPLANTATION
}

\section{Alemtuzumab induction reduces acute rejection risk}

Transplant rejection remains a serious and ongoing risk amongst kidney transplant recipients. The early findings of the $3 \mathrm{C}$ Study revealed that induction immunosuppression with alemtuzumab (a humanized monoclonal antibody capable of substantial lymphocyte depletion), combined with minimal exposure to standard immunosuppressive treatments, significantly reduced the risk of acute kidney transplant rejection.

The trial comprised 852 participants who were due to receive a kidney transplant within $24 \mathrm{~h}$, and assessed the efficacy of induction with alemtuzumab plus low-dose tacrolimus, mycophenolate mofetil and steroid avoidance, compared with the standard treatment regimen of basiliximab (an antiIL-2R antibody), standard-dose tacrolimus, mycophenolate mofetil and prednisolone. Excitingly, transplant recipients receiving alemtuzumab-based induction treatment had a $58 \%$ reduction in the risk of biopsy proven acute rejection compared with basiliximab-based treatment. This decrease in acute rejection was apparent within 1 month of transplantation. Unexpectedly, the researchers detected a greater effect on cellular-mediated than on antibody-mediated rejection.

Previous trials investigating alemtuzumab have shown a reduction in acute rejection when used in combination with a standard treatment regimen. However, this is the first trial to demonstrate positive effects of alemtuzumab with minimal exposure to standard treatments. The positive acute effects of alemtuzumab-based induction were without any significant increases in graft failure over 6 months or overall infections. However, a significantly greater risk of leukopenia and a small, but significant, increase in $\mathrm{BK}$ polyomavirus infection risk was evident in the alemtuzumab group.

Despite advances in induction and immunosuppression strategies, the long-term rates of transplant failure have remained static for 20 years. The primary aim of the $3 \mathrm{C}$ trial is to investigate the long-term effects of calcineurin inhibition on transplant failure. An accompanying editorial states that the second phase of the $3 \mathrm{C}$ trial involves re-randomization; recipients will receive maintenance immunosuppression with tacrolimus or sirolimus with mycophenolate mofetil, avoiding long-term exposure to calcineurin inhibitors in half of all transplant recipients.

The editorial notes that whilst the efficacy of conversion from tacrolimus to sirolimus is welldocumented, long-term effects of alemtuzumab induction therapy are not. Results from the 5-year, long-term follow-up of transplant recipients in the $3 \mathrm{C}$ study are, therefore, eagerly awaited.

\section{Peter Sidaway}

Original article The 3C Study Collaborative
Group. Alemtuzumab-based induction
treatment versus basiliximab-based
induction treatment in kidney transplantation
(the 3C Study): a randomised trial. Lancet
doi:10.1016/S0140-6736(14)61095-3
Further reading Kuypers, D. R. J.
Alemtuzumab induction therapy in kidney
transplantation. Lancet doi:10.1016/S0140-
6736(14)61174-0

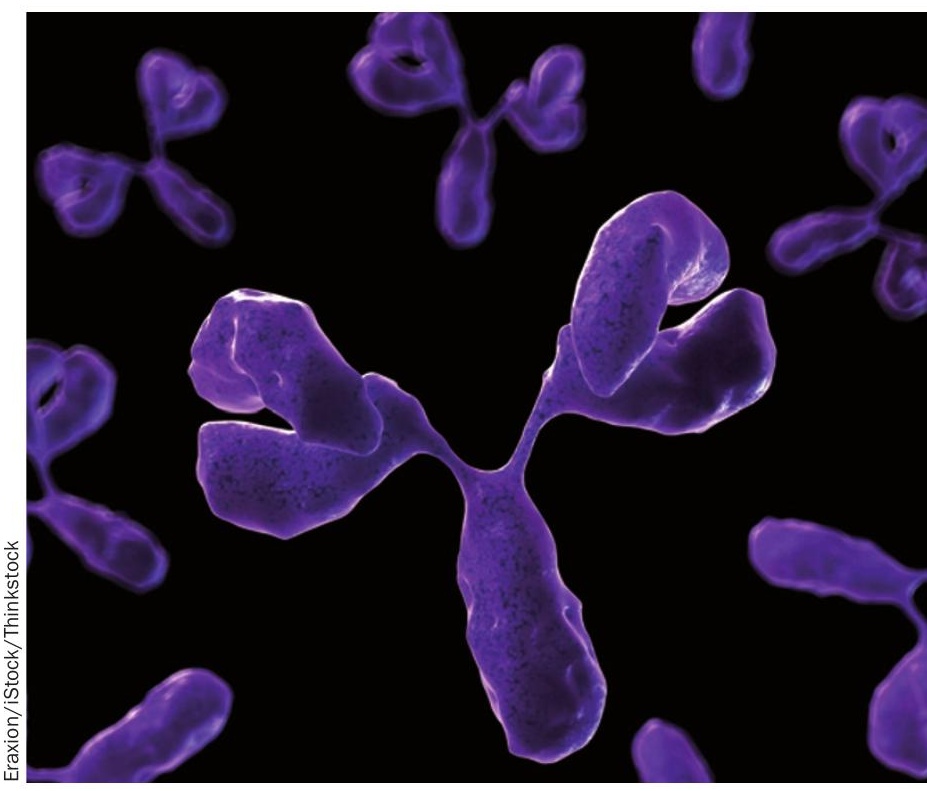

